\title{
BAUMWOLLE: GEFÜGE MIT GEWALT
}

Die Mikropolitik der Medien kann nicht «entdeckt» werden. Sie muss hergestellt und denkbar gemacht werden. Überall da, wo Medien neue Verflechtungen ermöglichen und neue Assoziationen evozieren, aber auch dort, wo neue Verkettungen von sozialen Ordnungssystemen, Technologien und Körpern neue Medien hervorbringen, lohnt es sich, diese Herstellung probeweise durchzuführen.

ANDREA SEIER ${ }^{1}$

Baumwolle umgibt uns in der Kleidung, im Kaffeepad am Morgen, in Geldscheinen und vielen anderen Materialverbünden. Die Entwicklung des Kapitalismus, die extreme Akkumulation von Kapital im Globalen Norden basiert nicht nur auf der kolonialen Landnahme und der Ausbeutung von Ressourcen, sondern ebenso (und in der Ausbeute vervielfacht) in der Vernutzung von, formal gesprochen, Arbeitskraft (von Körpern, von Leben). Exponentielle Profitsteigerungen werden durch den Anbau von Luxusgütern wie Zucker, Tabak und besonders Baumwolle erzielt. Anbau, Ernte und Verarbeitung dieser Stoffe brauchen nicht nur Landflächen, sondern erhebliche menschliche Arbeitskraft, ab dem Ende des I9. Jahrhunderts im Verbund mit Maschinen. Zusammen bilden sie eine «Plantagenmaschinerie $»^{2}$ oder - mit einem Begriff von Donna J. Haraway und Anna Lowenhaupt Tsing gesprochen - die «Plantationocene». ${ }^{3}$ Die Baumwolle ist darin nicht nur deshalb von Interesse, weil sich die westlichen Wissenschaften allgemein fragen müssen, welche Konsequenzen sie aus der kolonialen Geschichte ziehen. Sie gibt auch Figuren und Theoreme neu zu denken auf, mit denen die Medienwissenschaft arbeitet, wie etwa solche zu Materie/Medium und Form. Baumwolle kann so als ein Beispiel für die Verwobenheit von methodologischen und materiellen Fachbezügen in ein historisch-präsentes Gefüge von Techniken, Übersetzungen, Denken, Gewaltverhältnissen dienen. ${ }^{4}$

\footnotetext{
1 Andrea Seier: Mikropolitik der Medien, Berlin 2019, 25.

2 Eine Kombination von industrieller Landwirtschaft, neuartiger Arbeitsorganisation, Sklaverei und Landraub. Sven Beckert, Mindi Schneider: Der große Landraub, in: Die Zeit, Nr. 37, 5.9.2018, www.zeit. de/2018/37/nord-sued-konflikt-arbeits kraft-rohstoffe-kolonialzeit-ungleichheit (7.12.2019).

3 Donna Haraway: Anthropocene, Capitalocene, Plantationocene, Chthulucene: Making Kin, in: Environmental Humanities, Jg. 6, Nr. 1, 2015, 159-165, hier 16o; dies., Unruhig bleiben. Die Verwandtschaft der Arten im Chthuluzän, Frankfurt/M. 2018; Anna Tsing: Unruly Edges: Mushrooms as Companion Species, for Donna Haraway, in: Environmental Humanities, Jg. 1, Nr. 1, 2012, 141-154; dies.: Der Pilz am Ende der Welt. Über das Leben in den Ruinen des Kapitalismus, Berlin 2018.

4 Für kritische und produktive Kommentare danke ich herzlich Katrin Köppert und Naomie Gramlich.
} 
5 Sven Beckert zeichnet das seit dem 4. Jahrhundert in Indien und Mesoamerika und ab 950 in Europa nach. Ders.: King Cotton. Eine Geschichte des globalen Kapitalismus, München 2015, 31-39.

6 Ebd., 49.

7 Die Zahlen beziehen sich auf die Karibik, Süd- und Nordamerika. Marcus Rediker: The Slave Ship. A Human History, London 2007, 5 . Vgl. Michael Zeuske: Sklavenhändler, Negreros und Antlantikkreolen. Eine Weltgeschichte des Sklavenhandels im atlantischen Raum, Berlin, Boston 2015, 19.

8 Zur innerafrikanischen, arabischen und vorkolonialen Sklaverei vgl. Suzanne Miers, Igor Kopytoff (Hg.): Slavery in Africa. Historical and Anthropological Perspectives, Madison 1977.

9 Ich spreche von «Sklaven〉 und nicht von ‘Sklav_innen o oder ‘Versklavten`, wenn ich mich auf die entsprechenden verdinglichenden und entmenschlichenden Praktiken beziehe, und nehme das affirmative Moment darin in Kauf.

10 Beckert: King Cotton, 47.

11 Ebd., 203-211.

12 Ebd., 214.

13 Ian Baucom: Specters of the Atlantic. Finance Capital, Slavery, and the Philosophy of History, Durham,

London 2005, 17 u. Part 1: «Now

Being: Slavery, Speculation, and the Measure of our Time».

14 Ebd., $61 \mathrm{f}$.

15 Ebd., 61; Beckert: King Cotton, $86 \mathrm{f}$., 92 et passim.

16 Katrin Köppert, persönliche Mitteilung, 15.12.2019.

17 Das Verhältnis von Form und Wert in diesem Kreditwesen sei ein hauntologisches, so Baucom: Specters of the Atlantic, 31 et passim. Vgl. auch Joseph Vogl: Gespenst des Kapitals, Zürich 2011.

18 Man erfährt z. B., dass der Preis eines Sklaven (21-38 Jahre) im Jahr 1804 noch 450 Dollar betrug, 1860 schon 1.200 Dollar. Man konnte Sklaven zu einem Zins von $8 \%$ kaufen und eine Verdreifachung seines Vermögens erwirtschaften. Banken verdienten daran.

\section{Spekulation und «collaterals»}

Baumwolle hat nicht nur eine Geschichte als Kleidungsstoff. Sie hat auch eine Geschichte, in der sie wie Geld funktioniert. Denn Baumwollwaren speichern Arbeitskraft. Sie sind eine wichtige Wertanlage und ein Tauschmittel. Herrscher auf der ganzen Welt forderten Steuern oder Abgaben in Form von Baumwollstoffen. Sie sind ein ideales Tauschmittel, denn anders als Rohbaumwolle kann man sie leicht über weite Strecken transportieren, sie sind widerstandsfähig, Kapital, ein Protogeld. Nahezu überall in der vormodernen Welt konnte man mit Stoff bezahlen, ${ }^{5}$ und «afrikanische Machthaber und Händler verlangten überwiegend Baumwollstoffe», auch für die im Inland gefangenen Menschen. ${ }^{6}$ Zwischen dem späten I 5. und dem Ende des I 9. Jahrhunderts wurden I 2,4 Millionen Afrikaner_innen entführt (zwei Drittel davon zwischen I 700 und I 808). Wie viele Menschen auf dem innerafrikanischen Weg zu den Schiffen starben, ist nicht bekannt. Forscher_innen gehen von Zahlen zwischen einem Zehntel und der Hälfte aller Gefangenen aus; eine konservative Schätzung von I 5 Prozent ergibt weitere I, 8 Millionen Tote in Afrika. Auch während der Middle Passage starben I,8 Millionen. ı,6 Millionen Überlebende der Überfahrt wurden Zwangsarbeiter_innen, meist auf Plantagen. Weitere I 5 Prozent (oder mehr) starben im ersten Jahr der Zwangsarbeit. I4 Millionen Menschen wurden versklavt, und 9 Millionen Zwangsarbeiter_innen überlebten. ${ }^{7}$

Oft heißt es zur Relativierung der kolonialen Gewaltverbrechen, insbesondere der Versklavung, es habe Sklaverei ja schon in vorkolonialer Zeit gegeben. ${ }^{8}$ Aber im transatlantischen Dreieckshandel wurde der versklavte Mensch in einem vorher nicht gekannten Ausmaß zum Ding, zur Ware. Diese Dingwerdung ging weitgehend ungedachte Verbindungen mit der Ware Baumwolle ein. Im I9. Jahrhundert war die Verbindung von Sklaverei und Baumwolle unauflösbar. ${ }^{9}$ Profitable Verkettungen wurden durch Techniken des Überspannens von Zeit und Raum möglich: Um mehr Menschen zu kaufen, muss mehr Baumwolle angebaut werden, um für diejenigen zu zahlen, die dann wiederum u. a. Baumwolle anbauen. ${ }^{10}$ Und die Verflechtungen gehen weiter, denn es blieb nicht bei einfachen Entleerungen von Werten, Ersetzungen oder Geldäquivalenten. Baumwolle und Versklavte wurden Teil eines weiteren spins, der Entwicklung des Finanzwesens, genauer: des globalen transnationalen Kreditwesens. Neue Logistiken entstanden, etwa Formalisierungen von Materialproben, Standards im Vokabular und Messungen, Instrumente wie der Terminhandel, Frachtbriefe, Kommissionshandel, Vorschüsse und Kredite." Ohne Kredit wären die Baumwollimperien zusammengebrochen, es waren im Kern Kreditimperien. Die Kredite wurden abgesichert entweder durch die zukünftige Lieferung von Rohstoffen, die von Versklavten angebaut wurden, oder durch den Wert der Versklavten selbst. ${ }^{12}$ In dieser Betrachtung geht der Wert dem Tausch voraus, anders als bei Marx, wo Geld zunächst das leere Äquivalent sein muss, 
bevor getauscht wird. Hier wird kein Wert geschaffen, sondern er existiert bereits, genauer: Sobald ein Vertrag unterzeichnet ist, wird ein Wert existiert haben. ${ }^{13}$ Sklaven wurden nicht nur wie ein Typus einer Ware, sondern als ein Typus von zinsträchtigem Kapital behandelt. Sie waren nicht nur Gegenwert von einem Kaufwert, sondern Speicher für ein ganzes Kreditwesen, gleichzeitig selbst Ware und Depot eines dezentralen transatlantischen Banksystems, ein Pfand (deposit), das sofort in einen Schuldschein (bond) übergehen kann. Das, so Ian Baucom, ist so obszön wie zentral für diese Kapitallogik, und es ist nötig zu verstehen, wie hier nicht nur Menschen als Warentyp gehandelt werden konnten, sondern als flexible, verhandelbare, übertragbare Form von Geld. ${ }^{14}$ So entsteht Eigentum aus Zukunft. ${ }^{15}$ Allerdings, darauf hat Katrin Köppert hingewiesen, ist diese Idee von <Zukunft> positioniert, es handelt sich gerade nicht um die Zukunft der versklavten Menschen, sondern um die Zeit der Akkumulatoren im Globalen Norden. ${ }^{16}$ Auch das ist, was Baucom $\ll$ Specters of the Atlantic» genannt hat. ${ }^{17}$ Sklaven waren eine finanzielle $<$ Sicherheit $>$ (collateral), ein Gegenwert, Pfand für Kredite verschiedenster Sorten, in den Südstaaten, an der Wall Street, in Einzelhaushalten. ${ }^{18}$ Im Rahmen von «The I6r9 Project» der New York Times hat Matthew Desmond den gegenwärtigen Reichtum und seine Verteilung ökonomisch von der Sklaverei hergeleitet und bis in einzelne finanzökonomische Instrumente hinein verfolgt. ${ }^{19}$

Dies ist nur ein kleiner Ausschnitt aus einer Wirtschaftsgeschichte, die die Materialität nicht nur von Baumwolle, sondern auch von zahlreichen anderen Rohstoffen in eins gewoben hat mit Maschinen, Arbeit, Logistik und Menschen - Grundlage für heutige strukturelle Ungleichheit, Grundlage für Reparationsforderungen in den USA und in Großbritannien ${ }^{20}$ und auch Grundlage für ein medienhistorisches Novum, das der Entwicklung von Notationssystemen für die Buchhaltung. Diese beruhten auf Überwachungsmethoden, Menschenvermessung, Verbuchungsmethoden körperlicher Ressourcen und einem mathematisch ausgeklügelten Foltersystem, wie Julia Ott berichtet. Aufseher wogen am Ende jedes Arbeitstages die von jedem_jeder einzelnen Arbeiter_in gepflückte Baumwollmenge und notierten sie mit Kreide auf einer Tafel. Das Ergebnis wurde mit der individualisierten Quote jedes_jeder einzelnen Arbeiters_in verglichen. Wer weniger gepflückt hatte als am Vortrag, wurde der Differenz entsprechend ausgepeitscht. Neue Quoten wurden errechnet und die Tafel gesäubert. Die Quoten stiegen unaufhörlich. Die Produktionssteigerung zwischen I 800 und I 860 lieferte eine ebensolche Steigerung der Erträge, die die neuen Maschinen und Spinnereien in Manchester verarbeiten konnten. ${ }^{21}$

Stefano Harney und Fred Moten führen in Die Undercommons ${ }^{22}$ den Kapitalismus nicht auf die Lohnarbeit, sondern die Sklaverei zurück und damit, hauptsächlich, auf die Logik und Logistik der Middle Passage seit dem I 5 . Jahrhundert. Sie analysieren weniger die Industrialisierung als den Kolonialismus, weniger die Produktion als die Zirkulation von Waren. Diese ist bestimmt vom vorausgreifenden Zugriff auf Optionen, geschildert als eine gewaltsame
19 Das Projekt entwirft die Geschichte der USA neu und geht dabei nicht von deren ‘Gründung, 1776 , sondern von der Ankunft der ersten ersten Sklav_innen 1619 aus. Matthew Desmond: In order to understand the brutality of American capitalism, you have to start on the plantation, in: The New York Times Magazine (The ${ }_{1619}$ Project), 14.8.2019, nyti.ms/2OVcThK (17.2.2020).

20 Kehinde Andrews: Reparationen wurden an die Sklavenbesitzer gezahlt; Versklavte erhielten nichts und gerieten durch ausbeuterische Lohnarbeit bei den vorigen Sklavenhaltern in neue Abhängigkeiten; $\mathrm{Na}$ tionen, die von den Kolonialmächten in die «Unabhängigkeit` entlassen wurden, wurden gezwungen, für die Hinterlassenschaften der Kolonialstaaten riesige Summen zu zahlen, was sie teilweise bis heute tun. Ders.: The west's wealth is based on slavery. Reparations should be paid, in: The Guardian, 28.8.2017, www. theguardian.com/commentisfree/2017/ aug/28/slavery-reparations-westwealth-equality-world-race (7.12.2019). Wenn man nur die Arbeitslöhne Versklavter in den USA berechnen würde, käme man auf heutige 6-14 Billionen US-Dollar. David Olusoga: Ab 1834 wurden an 46.00o Brit_innen Kompensationen in Höhe von heute 17 Billionen Pfund ausgezahlt (refinanziert durch die erhöhte Mehrwertsteuer, die wiederum die Armen überproportional traf; Sklaven mussten noch Jahre unbezahlt weiterarbeiten, solche im Haus vier Jahre, Feldarbeiter sechs), ders.: Britain's Forgotten Slave Owners, in: The Price of Freedom (1. Staffel, 2. Folge), BBC, GB 2015, www.youtube.com/ watch?v=jmmjGrUKnYI (7.12.2019). Siehe auch Thomas Craemer: Estimating Slavery Reparations: Present Value Comparisons of Historical Multigenerational Reparations Policies, in: Social Science Quaterly, Bd. 96, Nr. 2, 2015, 639-655.

21 Julia Ott: Slaves: The Capital that Made Capitalism, in: Public Seminar, 9.4.2014, www.publicseminar. org/2014/04/slavery-the-capital-thatmade-capitalism/ (7.12.2019).

22 Stefano Harney, Fred Moten: Die Undercommons. Flüchtige Planung und schwarzes Studium, Wien 2016. 
23 «Von hier aus entfalten sie [Harney/Moten] die historische Analyse eines Logistikregimes, das von allseitiger Kalkulation, Vorausberechnung und Prognostik zukünftiger Optionen bestimmt ist. Seine Gewalt liegt in der Beschlagnahmung der gesamten noch nicht individuierten, nicht verkörperten, nicht vergegenständlichten Zukunft, deren Offenheit in einer reflexiven, zunehmend algorithmisch gesteuerten Zirkulation von Güter-, Datenund Finanzströmen verschlossen wird.» Ciğdem Inan: Nichtanerkennung. Die andere Seite der Rassismuskritik, in: Texte zur Kunst, Nr. 113, Mai 2019, 74-91, hier 88.

24 Es geht um Versklavte, «hinter denen die Logistik simmer her ist, ohne sie vollständig aneignen oder regieren zu können: ‘Die Logistik konnte nicht halten, was sie in den Laderaum verbannt hatte.) Der Unterschied zwischen Ding und Objekt ist der zwischen bloßer Potenzialität (die sich in Bewegung befindet) und kolonialkapitalistisch bestimmter Ware (die transportiert wird).» Ebd. $88 \mathrm{f}$., Zitate im Zitat: Harney, Moten: Die Undercommons.

25 Friederike Gesing u. a.: NaturenKulturen-Forschung. Eine Einleitung, in: dies. u. a. (Hg.): NaturenKulturen. Denkräume und Werkzeuge für neue politische Ökologien, Bielefeld 2018, 7-50.

26 Tsing: Unruly Edges, 148.

27 Stoff, in: Deutsches Wörterbuch von Jacob und Wilhelm Grimm [ab 1854], Bd. 19, Sp. 141, hdl.handle. net/11346/VFHZ (18.2.2020).

28 Die Erweiterung dieser Aufzählungen, die Vergrößerung der Akteur_innen im Netzwerk löst nicht das methodische Problem, erlaubt aber eher eine Reflexion der Forscher_innenposition und eine Verantwortung für die eigene Perspektive. Danke für den Hinweis an Tobias Matzner bei der GfM-Jahrestagung Medien-Materialitäten 2019 in Köln; vgl. zur Kritik an Bruno Latours Netzpolitik: Ulrike Bergermann: Kettenagenturen. Latours Fotografien, Brasilien 1991, in: Ilka Becker u. a. (Hg.): Fotografisches Handeln, Ilmtal-Weinstraße 2016, 160-181.
Beschlagnahmung der noch nicht materialisierten Zukunft, deren Offenheit durch die Kalkulationen verschlossen wird ${ }^{23}$ - aber nicht komplett, insofern man auch die Offenheit von Affekten, Widerstandsfähigkeit, agency auch der Versklavten mitdenken muss. ${ }^{24}$

Um nicht der Logik der Sklaverei und der Verdinglichung zu folgen und da die Baumwolle nicht ohne die Sklaverei, die Körper nicht ohne den Rohstoff und das Protogeld zu haben sind, gilt es, mit den relationalen Ontologien nicht vom Körper an sich, sondern vom Körper in Umwelt, Umweltmitkörper, NaturenKulturen $^{25}$ etc. zu sprechen, Figuren der Assemblage, des Gefüges, der AkteurNetzwerke zu borgen und in aller NatureCulture und CompanionSpecies wiederum nicht die Gewalt außen vor zu lassen (auch ANT und Relationale Ontologie müssen nach der Rassismusgeschichte ihrer Netzwerkknoten, den menschlichen wie nichtmenschlichen, fragen). Tsing hat in ihrer Geschichte der Plantage, dem Hybrid von Technologien, Pflanzen und Menschen, geschrieben: $\ll[\ldots]$ one ingredient is missing: They remove the love.» ${ }^{26}$ Beziehungen sind Teil von Gefügen, nicht nur Apparate, Menschen und Materialien; daher muss eine Netzwerk-Analyse mit Transmissionen, Affekten, Macht oder auch Liebe als eigenen Größen zu rechnen lernen.

\section{Stoff - Medium und Form, und Gewalt}

Im deutschen Wort <Stoff $>$ klingt eine Doppelbedeutung von Pflanzenteil, Textilrohstoff und bereits gewobenem Tuch an, von cotton und cloth. Es bezeichnete ursprünglich ein Seidengewebe und wurde um das Jahr Iooo auch als <Materie> im Gegensatz zur <Form> benutzt. ${ }^{27}$ Die Etymologie erinnert an kritische Lektüren der Materie/Form-Unterscheidung sowie der von Medium und Form. Baumwolle muss zunächst einmal als Rohstoff produziert werden - auf dem Feld ist sie als Teil einer Pflanze der klassischen Idee von Materie noch am nächsten, auch wenn argumentiert werden kann, dass die Baumwollkapsel nur in einem Milieu von Feld, Handarbeit, Gin (der Kapselentfernungsmaschine) und dem Rest der Verarbeitungskette Sinn macht, d.h. aus der Kombination von Baumwollballen, Sklavenkauf, Zeit, Muskelarbeit, biopolitischen Notationssystemen, Gewalt, Sonne und Wasser Wert entsteht. Die Faser hat keinen Sinn, sie hat keinen Wert, aber sie wird sich in diesem Gefüge auf vorher noch nicht dagewesene Weise in mehreren Punkten der Logistik wiederfinden (als Faden, als Tuch, als Geld). Die Materialität von Baumwolle tritt erst in Erscheinung, wenn sie als transformatives Ding, im Werden, im Verbund mit Schwarzen Händen, weißen Peitschen, britischer Maschinerie und Finanzwirtschaft steht. (Man könnte weiter die Schifffahrt, den Verweis auf jahrhundertealte Kulturtechniken, Witterungsbedingungen etc. hinzufügen. ${ }^{28}$ ) Ohne Logistik wird keine Materie produziert werden. Das platziert die Logistik nicht vor oder außerhalb der Materialität, sie ist vielmehr selbst materiell, wie analog zu Judith Butlers Körper von Gewicht und ihrer Zurückweisung 
der Annahme, die Performativität in der Hervorbringung von Körpern werde nur-diskursiv, also immateriell gedacht, formuliert werden kann. ${ }^{29}$ Diese führt Butler im close reading von Aristoteles (Materie als Potenzialität, Form als Aktualität) aus.

Stellen wir uns Baumwollfäden in einer Kapsel vor, isoliert: Das entspräche im Modell der byle, dem Holz, das Aristoteles der Form (morphe), die sich in sie eintragen wird, entgegengesetzt hat. (Dass hier von Holz und nicht vom Baum die Rede ist, verweist schon darauf, dass die ursprüngliche Materialität schwer zu haben ist - die Materie ist immer schon im Gefüge mit Blättern und Insekten [Holz] oder mit Samen, Fingern, Spinnrädern [Baumwolle] da.) Der Stuhl, der aus dem Holz werden wird bzw. diesem eine Form geben wird, entspräche dem gewebten Tuch, das aus den Pflanzenfäden werden wird. Die Fäden werden nicht ohne Arbeiter_innen, Webstuhl etc.; in dieser Gleichung stünden sie auf der Seite des passiven beschreibbaren/bedeutbaren Materials; als Element in einem performativen oder assemblierten Setting wären allerdings immer andere Aktualisierungen ihrer Potenzialität mitgedacht (durch biologische Mutationen, soziale Meuterei, agency der Versklavten, finanztechnischen Börsencrash o. Ä.). In die Geschichte jeder Materie sind die Effekte der Macht eingeschrieben. Das gilt für byle/materia ebenso wie für Medium und Form.

Die medienwissenschaftliche Diskussion dieser Unterscheidung schien zunächst ganz nach Luhmann die Machtfrage zu suspendieren. Leander Scholz hat die Bestimmung von Medium und Form auf in die Medienwissenschaft mitgenommene Zuschreibungen von Passivität bzw. Aktivität von $<$ Medien $>$ verfolgt.

In der philosophischen Tradition von Descartes bis Luhmann lässt sich ein Denken des Medialen beobachten, das die spezifische Eigenschaft dessen, was überhaupt ein Medium sein kann, gerade darin sieht, dass seine tendenzielle Eigenschaftslosigkeit es zu einem hervorragenden Träger von Einschreibungen macht. Das Mediale erscheint dabei als eine passive Unterlage, die nur im Gegensatz zu einem Ding oder einer Form selbst thematisch werden kann. [...] Die Aufmerksamkeit dieses Denkens richtet sich deshalb auf die Differenz von Ding und Medium oder von Form und Medium als Differenz zwischen einer beschreibbaren Gestalt und einer sich im Hintergrund dieser Gestalt zeigenden Gestaltlosigkeit. Während eine Form oder ein Ding fest umrissene Grenzen besitzt, ist das Mediale demnach durch einen Zustand der Latenz und der Potenzialität gekennzeichnet und deshalb grundsätzlich von der Seinsweise eines Dings oder der Beobachtbarkeit einer Form unterschieden. Zugleich aber scheint der hyletischen Passivität des so verstandenen Medialen immer schon ein Begehren innezuwohnen, aufgrund dessen sich das Medium stets auf dem Weg zu seiner <Beseelung> durch die aktive Form befindet. $^{30}$

Was ein Medium sein kann, wird innerhalb dieses Paradigmas stets von der Seite der Form aus gedacht. Von der Potenzialität der Form auszugehen, wiederholt allerdings einen Dualismus von aktiv und passiv, so Scholz,
29 Judith Butler: Körper von Gewicht. Die diskursiven Grenzen des Geschlechts, Frankfurt/M. 1997, bes. 22, 32, 55, 58 et passim. "Die Materie der Körper wird neu gefaßt als die Wirkung einer Machtdynamik, so daß die Materie der Körper nicht zu trennen sein wird von den regulierenden Normen, die ihre Materialisierung beherrschen, und von der Signifikation dieser materiellen Wirkungen.» (Ebd. 22) "Es ist von Anfang an klar, daß Materie eine Geschichte hat (sogar mehr als eine) und daß die Geschichte der Materie zum Teil bestimmt ist von der Aushandlung der sexuellen Differenz.» (Ebd. 55)

30 Leander Scholz: Rasterfahndung oder Wie wird Wachs gemacht, in: Jens Schröter, Alexander Böhnke (Hg.): Analog/Digital - Opposition oder Kontinuum? Zur Theorie und Geschichte einer Unterscheidung, Bielefeld 2004, 97-116. Scholz beendet seine Lektüre mit einer kritischen Einschätzung: «Was überhaupt ein Medium sein kann, wird deswegen nach wie vor allein von der Formseite her gedacht, was zur Folge hat, dass die traditionellen Zuschreibungen von passiv (Medium) und aktiv (Form) letztlich unangetastet bleiben. Dass das Medium sich nicht abnutzt oder verbraucht und zugleich jenseits seiner Informierung durch die Form zum «Nichts tendiert, also keine Widerständigkeit besitzt, ist daher nur die andere Seite dieses einschränkenden Weltbegriffs und macht deutlich, dass bei der so getroffenen Unterscheidung von Medium und Form die Formseite zwar die Medienseite informiert, die Formseite von der Medienseite aber unbehelligt bleibt.» (Ebd., 99) Danke für den Hinweis an Thomas Waitz. 
31 Friedrich Balke, Leander Scholz: Das Medium als Form, in: Transkriptionen, Nr. 3, 2004, 2-7, hier 4 .

32 Haraway: Anthropocene, Capitalocene, Plantationocene, Chthulucene, 160.

33 «[...] the slave plantation system was the model and motor for the carbon-greedy machine-based factory system that is often cited as an inflection point for the Anthropocene.» (Ebd.) «Während in Amerika Genozide an der amerikanischen Bevölkerung verübt wurden, wurden Millionen von Menschen aus afrikanischen Ländern in die Sklaverei verkauft - rassistisches Unrecht bildet die Basis dieses Wirtschaftssystems. Durch die Privatisierung von Allmendeland verlor die ärmere Landbevölkerung in Europa die Grundlagen für ihre Subsistenzproduktion. Diese Einhegungen schufen auch die Knappheit, die bis heute die Grundlage kapitalistischen Wachstums darstellt. Denn Menschen ohne Land für ihre Subsistenzproduktion waren zur Lohnarbeit gezwungen, auf der die produzierende Wirtschaft basiert - ein Prozess der ausgesprochen gewaltförmigen ‘ursprünglichen Akkumulation, der sich bis heute fortsetzt.» Matthias Schmelzer, Andrea Vetter: Degrowth/Postwachstum zur Einführung, Hamburg 2019, $48 f$.

34 Haraway: Unruhig bleiben, hier $80,75,10$.

35 «... skalieren heißt: etwas in Maßstäben so wachsen oder schrumpfen lassen, dass es zirkulieren kann, gehandelt werden kann, berechnet werden kann, zu einer Ware werden kann, die ihren Wert auch über Grenzen hinweg darstellt»; diese Art von Abstraktionen will Tsing nicht mitvollziehen und sucht andere Übersetzungsketten im Pilzhandel. Tsing: Der Pilz am Ende der Welt, 45 u. $58 \mathrm{ff}$.

36 Ebd., 59-61.

37 Naomie Gramlich, persönliche Mitteilung, 15.12.2019.

38 Seier: Mikropolitik der Medien, 25 et passim. um <Medien> als Raum unbegrenzter Möglichkeiten zu bestimmen - mit all seinen genderings. Scholz und Friedrich Balke haben ihrer Kritik an einer <latenten Spiritualität $>$ Luhmanns eine Perspektive hinzugefügt, die die Debatte an Machtfragen anschließbar macht. Wo Luhmann den Medienbegriff von allen Konnotationen des <alten> Materiebegriffs fernhalten wollte, um ihn als einen Raum schier unbegrenzter Möglichkeiten zu begreifen, schlagen sie vor, mit Michel Foucault nicht von einer Möglichkeits-, sondern von einer Existenzfunktion auszugehen, «die dafür sorgt, dass aus einer offenen Mehrheit möglicher Verbindungen nur bestimmte Strukturmuster ausgewählt und realisiert werden». ${ }^{31}$

\section{Responsable Wissensökonomien}

Wie die Realisierungen dann geschehen, ist jeweils am konkreten Fall zu analysieren, denn ihre Möglichkeiten sind nicht unbegrenzt; sie sind eingebettet in Umwelten, die wir methodologisch in anderen Fächern zu verorten gelernt haben. Kolonialität in Konzepten der Wirtschaftsgeschichte, der Sozialgeschichte, der Geschichte der Gewalt zu sehen, drängt sich nicht nur auf, sondern ist auch unhintergehbar, wie Haraway und Tsing mit ihren Gefügen von Materialität und Macht zu skizzieren begonnen haben. Haraway greift den 2014 in einem Gespräch an der Universität Aarhus entwickelten Begriff des plantationocene auf, um «multispecies assemblages» zu beschreiben: ${ }^{32}$ Das «Zeitalter der Plantage» basiert auf Sklavenarbeit, Landwirtschaft und Rohstoffextraktionen. Es wird Modell und Motor des <baumwoll-gierigen Fabriksystems〉 des Anthropozäns. ${ }^{33}$ Als weiteren Epochenbegriff ohne Epoche - nicht in Folge, sondern nebeneinander gedacht - entwirft Haraway das «Chthuluzän»: Es ist keine Geschichte, in der der Mensch (oder der Mensch mit Technik) im Mittelpunkt steht wie beim Anthropozän. Das Chthuluzän setzt sich «aus Fortsetzungsgeschichten und artübergreifenden Praktiken des Miteinander-Werdens zusammen», es ist post-eurozentrisch, «ein Zeitort des Lernens mit der Idee eines responsablen (response-able) gemeinsamen Lebens und Sterbens auf einer beschädigten Erde». ${ }^{34}$ Und mit ihrer Idee von Humus korrespondiert ein modellhaftes Pilzgefüge: Anna Tsing hat in Der Pilz am Ende der Welt ein Gefüge von Natur, Kultur, Techniken und Wertbestimmungen rund um den Matsutakepilz geschrieben; in ihrer Suche nach «kontaminierter Diversität» und einer «Theorie der Nichtskalierbarkeit» beschreibt sie die Plantage als das Gegenteil der angestrebten Ökonomie. ${ }^{35}$ Skalierbarkeit wird sichtbar als formaler Grundbaustein einer kapitalistischen Maschinerie, die nicht nur Austauschbarkeit und Mechanisierung, sondern auch Wiederholbarkeit von Prozessen in verschiedenen Maßstäben und auf Expansion zielend vorsieht. Das <Material> unterliegt der Isolierung, die Teile können sich nicht mehr austauschen, nicht mehr reproduzieren, Zuckerrohrsetzlinge werden zu Klonen, Versklavte voneinander getrennt und entfremdet: eine Fabrikbarmachung austauschbarer 
und kontrollierter Einheiten. Die Welt als Fabrik wird im Modus der Plantage bewirtschaftet. ${ }^{36}$ Naomie Gramlich hat darauf hingewiesen, dass solche Analogien allerdings wiederum in Gefahr stehen, universalistisch zu argumentieren, insofern sie in ihrer «Gleichmachung der Menschen, des Bodens und der Baumwollpflanzen blind sind gegenüber den Aufständen der Maroons, Kulturformen wie dem Blues oder auch den Sklav_innengärten, die alle Beiwerke der Plantage sind - dabei wären das genau Haraways sym-Geschichten des Chthuluzäns».37 Die Welt der Wissenschaft sucht andere Ökonomien; die Materialität namens Baumwolle wird in ihrem analytischen Blick nicht mehr isoliert Sinn machen, sondern als Teil einer Szene, eines -zän. Anders als in den vorigen zitierten Perspektiven spielt bei Haraway und Tsing die Zeit - als eine fortschreitende Zeit - keine große Rolle. Ihre -zäne folgen nicht aufeinander, gehen nicht selbst Wetten auf die (wissenschaftliche) Zukunft ein.

Mit Andrea Seier sind diese Wissensformen und Materialitäten nicht einfach aufzufinden, sondern sie werden im Zuge ihrer Suche denkbar gemacht. ${ }^{38}$ Auch Baumwolle ist Ergebnis und Ausgangspunkt von As-Soziationen greifbarer, logistischer, ideologischer Elemente. Und die Materialität der Baumwolle ist in weitere Ökonomien eingewoben. Die Droge Baumwolle erzeugte «starke halluzinatorische Träume von territorialer Expansion». ${ }^{39}$ Sie wurde das «Haschisch des Westens» genannt und reiht sich ein in die «drug foods» Tabak, Kaffee, Schokolade und Zucker, die Luxusgüter des I 7. Jahrhunderts, die eine «Kultur des Geschmacks» begründeten, über deren Ästhetik und Politik weiter zu sprechen ist. ${ }^{40}$ Der körperliche Suchtcharakter der kolonialen Waren verweist bis in die Gegenwart darauf, dass diese Logistik nicht ohne unsere Affekte zu denken und auszurechnen sein wird.
39 Abolitionist John Greenleaf Whittier zit. n.: Beckert: King Cotton, 232.

40 Simon Gikandi: Slavery and the Culture of Taste, Princeton 2011; Ruth Sonderegger mit Fragen von Mara Recklies: Fragen zur Kolonialität der europäischen Ästhetik, in: Eva Knopf, Sophie Lembcke, Mara Recklies (Hg.): Archive dekolonisieren. Mediale und epistemische Transformationen in Kunst, Design und Film, Bielefeld 2018, 251-258; vgl. auch Iris Därmann: Landnahme, Menschennahme. John Locke und der transatlantische Sklavenhandel, in: Volker Gottowik, Holger Jebens, Editha Platte (Hg.): Zwischen Aneignung und Verfremdung. Ethnologische Gratwanderungen. Festschrift für KarlHeinz Kohl, Frankfurt / M., New York 2009, 69-82; dies.: "Stealing away». Trauer und suizidale Melancholie im transatlantischen Sklavenhandel, in: Zeitschrift für Ästhetik und allgemeine Kunstwissenschaft, 2018, 41-59; Katja Diefenbach: Besitzindividualismus und transatlantische Sklaverei im Spiegel der frühmodernen Philosophie, in: Die Bestie und der Souverän/The Beast and the Souvereign, Katalog, Württembergischer Kunstverein Stuttgart/MACBA Museu d'Art Contemporal de Barcelona, hg. v. Hans D. Christ u. a., Leipzig 2018, 154-175. 